\title{
Effects of online hemodiafiltration on anemia and nutritional status in chronic hemodialysis patients
}

\author{
Yu Ho Lee ${ }^{1, *(\mathbb{D})}$, Yoon Soo Shin ${ }^{1, *(\mathbb{D})}$, So-Young Lee ${ }^{1(\mathbb{D}}$, Yang Gyun Kim ${ }^{2(\mathbb{D}}$, Sang Ho Lee ${ }^{2(\mathbb{D})}$, Ju Young Moon ${ }^{2(\mathbb{D})}$, \\ Kyung Hwan Jeong ${ }^{3(\mathbb{D}}$, Hyeon Seok Hwang ${ }^{3(\mathbb{D}}$, Shin Young Ahn ${ }^{4(\mathbb{D})}$, Hong Joo Lee ${ }^{5(\mathbb{D})}$, Dong-Young Lee ${ }^{6(\mathbb{D})}$,

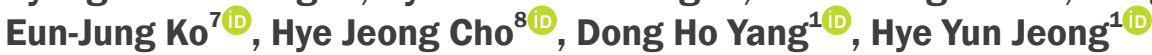 \\ ${ }^{1}$ Division of Nephrology, Department of Internal Medicine, CHA Bundang Medical Center, CHA University School of Medicine, Seongnam, \\ Republic of Korea \\ ${ }^{2}$ Division of Nephrology, Department of Internal Medicine, Kyung Hee University Hospital at Gangdong, College of Medicine, Kyung \\ Hee University, Seoul, Republic of Korea \\ ${ }^{3}$ Division of Nephrology, Department of Internal Medicine, College of Medicine, Kyung Hee University, Seoul, Republic of Korea \\ ${ }^{4}$ Division of Nephrology, Department of Internal Medicine, College of Medicine, Korea University, Seoul, Republic of Korea \\ ${ }^{5}$ Division of Nephrology, Department of Internal Medicine, Seoul Red Cross Hospital, Seoul, Republic of Korea \\ ${ }^{6}$ Division of Nephrology, Department of Internal Medicine, VHS Medical Center, Seoul, Republic of Korea \\ ${ }^{7}$ Department of Internal Medicine, Sa-lang Hospital, Ansan, Republic of Korea \\ ${ }^{8}$ Division of Nephrology, Department of Internal Medicine, International Naeun Hospital, Anyang, Republic of Korea
}

\begin{abstract}
Background: Online hemodiafiltration (OL-HDF) offers considerable advantages in clearance of molecules of various sizes. However, evidence of clinical effects of OL-HDF is scarce in Korea. In this study, we investigated changes in laboratory values over more than 12 months after switching to OL-HDF.

Methods: Adult patients with end-stage renal disease undergoing hemodialysis (HD) were prospectively enrolled in a K-cohort (CRIS no. KCT0003281) from 6 tertiary hospitals in South Korea. We recruited 435 patients, 339 of whom were on HD at enrollment. One hundred eighty-two patients were followed for more than 24 months. Among them, 44 were switched to OL-HDF for more than 12 months without conversion to HD. We used a paired $t$ test to compare baseline and 24-month follow-up results.

Results: The mean age of the subjects was $61.2 \pm 12.2$ years, and $62.6 \%$ were male. The baseline hemoglobin level was not significantly different between HD and OL-HDF group $(10.61 \pm 1.15$ vs. $10.46 \pm 1.03 \mathrm{~g} / \mathrm{dL}, P=0.437)$. However, the baseline serum protein and albumin levels were significantly lower in the OL-HDF group $(6.82 \pm 0.49$ vs. $6.59 \pm 0.48 \mathrm{~g} / \mathrm{dL}, P=0.006 ; 3.93 \pm 0.28$ vs. $3.73 \pm 0.29 \mathrm{~g} / \mathrm{dL}, P<0.001)$. In patients switched to OL-HDF, levels of hemoglobin and serum albumin significantly increased $(10.46 \pm 1.03$ vs. $11.08 \pm 0.82 \mathrm{~g} / \mathrm{dL}, P=0.001 ; 3.73 \pm 0.29$ vs.
\end{abstract}

Received August 1, 2019; Revised December 4, 2019; Accepted December 4, 2019

Edited by Young-Ki Lee, Hallym University, Seoul, Republic of Korea

Correspondence:

Hye Yun Jeong

Division of Nephrology, Department of Internal Medicine, CHA Bundang Medical Center, CHA University School of Medicine, 59 Yatap-ro, Bundang-gu, Seongnam 13496, Republic of Korea. E-mail: heun-j@hanmail.net

Dong Ho Yang

Division of Nephrology, Department of Internal Medicine, CHA Bundang Medical Center, CHA University School of Medicine, 59 Yatap-ro, Bundang-gu, Seongnam 13496, Republic of Korea. E-mail: dhyang@cha.ac.kr

*Yu Ho Lee and Yoon Soo Shin contributed equally to the work as co-first authors.

Copyright (C) 2020 by The Korean Society of Nephrology

(a) This is an open-access article distributed under the terms of the Creative Commons Attribution Non-Commercial License (http://creativecommons.org/ licenses/by-nc-nd/4.0/), which permits unrestricted non-commercial use, distribution, and reproduction in any medium, provided the original work is properly cited. 
$3.87 \pm 0.30 \mathrm{~g} / \mathrm{dL}, P=0.001)$. The normalized protein catabolic rate decreased after 24 months, but the change was not significant $(1.07 \pm 0.25$ vs. $1.03 \pm 0.21 \mathrm{~g} / \mathrm{kg} /$ day, $P=0.433)$. Although the dose of erythropoiesis-stimulating agent was lower in patients who converted to HDF, it was not significantly different $(-115.7 \pm 189.7$ vs. $-170.5 \pm$ $257.1 P=0.206$ ).

Conclusion: OL-HDF treatment over more than 12 months was associated with no harmful effects on anemia and nutritional status.

Keywords: Anemia, Dialysis, Kidney failure, chronic, Hemodiafiltration, Nutritional status

\section{Introduction}

The global prevalence and incidence of maintenance dialysis are increasing [1,2]. World Bank and World Health Organization data show that, by 2030, 2.16 million people in Asia will need renal replacement therapy (RRT), an increase of 223\% [3]. Among the modalities of RRT, maintenance hemodialysis (HD) has been a major method worldwide for patients with end-stage renal disease (ESRD) $[2,4]$. According to a US Renal Data System report, 87.3\% of incident patients with ESRD in the US used HD for RRT in 2016. The recent introduction of online-hemodiafiltration (OL-HDF) may offer major advantages in clearing molecules of various sizes, reducing HD-associated amyloidosis and chronic inflammation [5-7]. Because this modality has been officially approved for ESRD patients, the number of patients worldwide undergoing OL-HDF therapy doubled between 2004 and 2010, reaching 80,000 [8]. Previous studies in Japan and Europe suggest that OLHDF treatments are associated with greater hemodynamic stability and survival compared with standard HD [6,810]. However, the recent Dialysis Outcomes and Practice Patterns Study, which employed a "real-world" setting for cohort patients, did not find superior survival with HDF compared with HD [11]. A recent randomized controlled study of 100 patients in the UK also failed to find a superior survival outcome with OL-HDF [12].

In Korea, the annual number of patients undergoing HD has grown continuously from 49,403 in 2008 to 77,933 in 2016, a 1.57-fold increase [13]. However, few studies have investigated the effects of switching to OLHDF on various parameters in Korean patients because the cost difference between conventional HD and HDF is not reimbursed in Korea. The aim of our study was to investigate changes in clinical and laboratory features in patients with ESRD who switched from HD to OL-HDF, using data from a K-cohort comprising prospectively enrolled patients from 6 dialysis centers in South Korea.

\section{Methods}

\section{Study population}

The present study included data from a K-cohort (CRIS no. KCT0003281) of prospectively enrolled adult patients with ESRD undergoing HD in 6 tertiary hospitals in South Korea. Criteria for inclusion were age older than 18 years and treatment with HD 3 times per week ( $\geq 12 \mathrm{~h} /$ week)

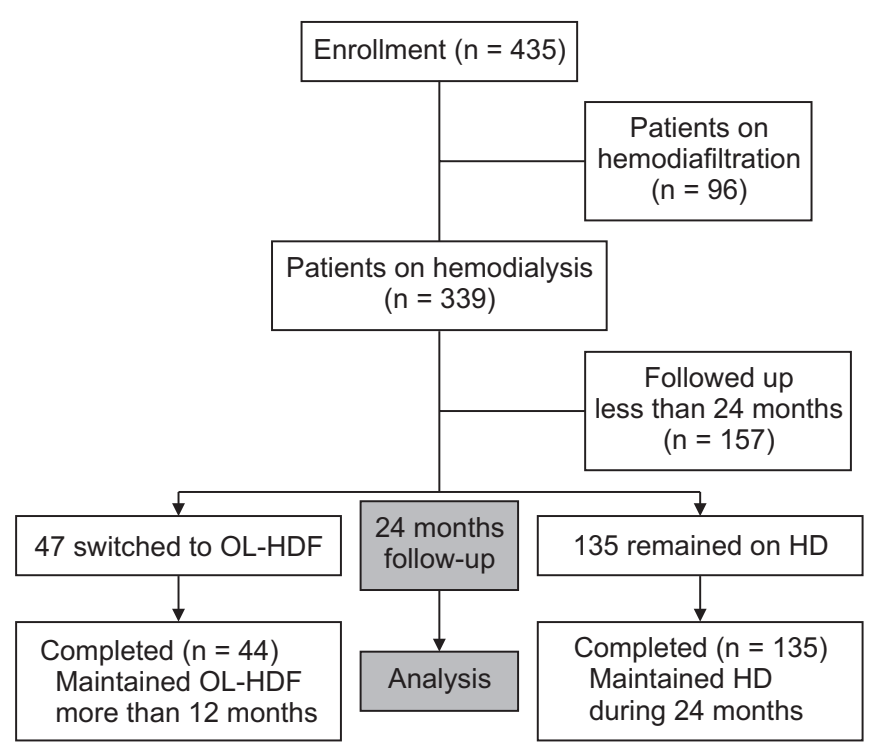

Figure 1. For this study, 435 patients were recruited, and 339 patients were on hemodialysis (HD) at the initial point. Among 339 patients on HD, 182 were followed up for more than 24 months, and 47 patients switched to hemodiafiltration (HDF) during the follow-up period. We investigated change in parameters in 44 patients maintained for more than 12 months after switching to Online HDF (OL$\mathrm{HDF}$ ) and in 135 patients who remained on HD during the follow-up period. 
for at least 3 months with no exposure history of renal transplantation. Patients with a history of cancer, coagulation disorders, or active infections were excluded.

A total of 435 patients was recruited, and 339 patients were on $\mathrm{HD}$ at the beginning of the study period. Among the 339 patients, 182 were followed up for more than 24 months. During the follow-up period, 135 patients remained on HD and 47 switched to HDF. However, 3 of the 47 who switched to HDF later returned to HD. We compared clinical parameters in 44 patients who switched to OL-HDF and 135 patients who remained on HD (Fig. 1). We used the term "HD group" for patients who remained on HD and "OL-HDF group" for patients who switched to and were maintained on OL-HDF.

The study was approved by the Institutional Review Board of CHA Bundang Medical Center and was conducted in accordance with the Declaration of Helsinki and the principles of Good Clinical Practice (CHAMC 2016-05-
064-024). Written informed consent was obtained from all patients.

\section{Clinical variables}

Patient demographic and clinical data of age, sex, etiology of ESRD (diabetes, hypertension, glomerulonephritis, polycystic kidney disease, or unknown), mean blood pressure, and body mass index were obtained from a medical record review. Cardiac diseases were defined as a medical history of angina pectoris, positive treadmill test, myocardial infarction, percutaneous transluminal coronary angioplasty, coronary artery bypass surgery, or congestive heart failure. Cerebrovascular diseases were defined as a medical history of stroke, transient ischemic attack, or intracranial hemorrhage. Data from laboratory findings were collected on blood hemoglobin ( $\mathrm{Hgb})$, blood urea nitrogen, creatinine, calcium, phosphate,

Table 1. Baseline characteristics of study participants

\begin{tabular}{|c|c|c|c|c|}
\hline Characteristic & Overall $(n=179)$ & HD group $(n=135)$ & OL-HDF group $(n=44)$ & $P$ value \\
\hline Male & $112(62.6)$ & $86(63.7)$ & $26(59.1)$ & 0.595 \\
\hline Age (yr) & $61.2 \pm 12.2$ & $61.1 \pm 11.8$ & $61.4 \pm 13.5$ & 0.877 \\
\hline HD duration (mo) & $2.9 \pm 4.4$ & $5.4 \pm 4.7$ & $3.5 \pm 3.0$ & 0.003 \\
\hline Diabetes & $79(44.1)$ & $64(47.4)$ & $15(34.1)$ & 0.095 \\
\hline Cardiac diseases & $24(13.4)$ & $16(11.9)$ & $8(18.2)$ & 0.204 \\
\hline Cerebrovascular diseases & $38(21.2)$ & $26(19.3)$ & $12(27.3)$ & 0.179 \\
\hline $25-\mathrm{OH}$ vitamin $\mathrm{D}(\mathrm{ng} / \mathrm{mL})$ & $18.16 \pm 10.48$ & $17.45 \pm 10.17$ & $20.34 \pm 11.22$ & 0.113 \\
\hline Hemoglobin (g/dL) & $10.57 \pm 1.12$ & $10.61 \pm 1.15$ & $10.46 \pm 1.03$ & 0.437 \\
\hline Protein $(\mathrm{g} / \mathrm{dL})$ & $6.77 \pm 0.50$ & $6.82 \pm 0.49$ & $6.59 \pm 0.48$ & 0.006 \\
\hline Albumin (g/dL) & $3.88 \pm 0.29$ & $3.93 \pm 0.28$ & $3.73 \pm 0.29$ & $<0.001$ \\
\hline Creatinine (mg/dL) & $9.63 \pm 2.63$ & $9.55 \pm 2.61$ & $9.88 \pm 2.71$ & 0.463 \\
\hline Calcium (mg/dL) & $8.62 \pm 0.81$ & $8.72 \pm 0.81$ & $8.32 \pm 0.75$ & 0.005 \\
\hline Phosphate (mg/dL) & $4.91 \pm 1.34$ & $4.88 \pm 1.35$ & $5.01 \pm 1.33$ & 0.560 \\
\hline iPTH (pg/dL) & $250.43 \pm 186.41$ & $234.24 \pm 186.61$ & $300.10 \pm 178.80$ & 0.041 \\
\hline Total cholesterol (mg/dL) & $138.02 \pm 28.54$ & $138.35 \pm 28.09$ & $137.02 \pm 30.18$ & 0.790 \\
\hline LDL cholesterol (mg/dL) & $76.66 \pm 23.47$ & $74.30 \pm 22.96$ & $83.89 \pm 23.84$ & 0.018 \\
\hline Beta2MG (mg/L) & $24.39 \pm 7.89$ & $24.17 \pm 7.96$ & $28.50 \pm 1.92$ & 0.158 \\
\hline Session length (hr) & $3.86 \pm 0.23$ & $3.84 \pm 0.24$ & $3.91 \pm 0.21$ & 0.072 \\
\hline URR & $72.25 \pm 6.43$ & $71.73 \pm 6.58$ & $73.83 \pm 5.76$ & 0.060 \\
\hline $\mathrm{Kt} / \mathrm{V}$ & $1.54 \pm 0.28$ & $1.53 \pm 0.27$ & $1.61 \pm 0.30$ & 0.098 \\
\hline Blood flow rate $(\mathrm{mL} / \mathrm{min})$ & $262.68 \pm 21.11$ & $260.96 \pm 21.44$ & $267.95 \pm 19.36$ & 0.056 \\
\hline \multicolumn{5}{|l|}{ OL-HDF modality } \\
\hline Pre-dilution & - & - & $4(9.1)$ & - \\
\hline Convection volume (L) & - & - & $20.4 \pm 7.7$ & - \\
\hline
\end{tabular}

Data are presented as number of patients (\%) or mean \pm standard variation.

Beta2MG, beta 2-microglobulin; HD, hemodialysis; iPTH, intact-parathyroid hormone; LDL, low-density lipoprotein; OL-HDF, online-hemodiafiltration; URR, urea reduction ratio. 
intact-parathyroid hormone (iPTH), glucose, uric acid, total cholesterol, low-density lipoprotein cholesterol, Creactive protein, 25 -hydroxyvitamin $\mathrm{D}$, and albumin at the time of patient enrollment.

\section{Statistical analysis}

Categorical variables were recorded as number and percentage. Continuous variables are presented as the mean \pm standard variation or median. Student's $t$ test and Mann-Whitney $U$ test were used to compare continuous variables. Categorical variables were compared using the $\chi^{2}$ test or Fisher's exact test. We used a paired $t$ test to compare baseline and 24-month follow-up results. To investigate possible confounding factors, we used a linear regression analysis with multiple adjustments for changes in various laboratory values. A $P$ value $<0.05$ was considered significant. Statistical analyses were performed using IBM SPSS Statistics for Windows (version 21; IBM Corp., Armonk, NY, USA).

\section{Results}

\section{Baseline laboratory findings of included patients}

Clinical and biochemical characteristics are shown in Table 1. Among 435 patients recruited between June 2016 and December 2018, 182 were followed for more than 24 months. During the follow-up period, 44 patients were switched to OL-HDF and kept on the treatment for more than 12 months, while 135 patients remained on HD.

The mean age of the study subjects was $61.2 \pm 12.2$ years, and $62.6 \%$ were male. The etiologies of ESRD were diabetic nephropathy (44.2\%), hypertension (24.6\%), glomerulonephritis (12.3\%), autosomal dominant polycystic kidney disease $(6.1 \%)$, and others (12.8\%). The duration of dialysis was $2.9 \pm 4.4$ months, and it was significantly longer in patients in the non-switched group ( $5.4 \pm 4.7$ vs. $3.5 \pm$ 3.0 months, $P=0.003)$. Serum protein and albumin levels were significantly lower in the OL-HDF group $(6.82 \pm 0.49$ vs. $6.59 \pm 0.5 \mathrm{~g} / \mathrm{dL}, P=0.006 ; 3.93 \pm 0.28$ vs. $3.73 \pm 0.29 \mathrm{~g} /$ $\mathrm{dL}, P<0.001)$. The iPTH level was significantly higher in the OL-HDF group $(234.24 \pm 186.61$ vs. $300.10 \pm 178.80$ $\mathrm{pg} / \mathrm{mL}, P=0.041)$, and the calcium level was significantly lower (8.72 \pm 0.81 vs. $8.32 \pm 0.75 \mathrm{mg} / \mathrm{dL}, P=0.005$; Table 1$)$.

\section{Effects of HDF on laboratory findings}

We compared baseline and 24-month follow-up laboratory results using a paired $t$ test. In the group that was switched from HD to OL-HDF, the levels of Hgb and serum albumin increased significantly (10.46 \pm 1.03 vs. $11.08 \pm 0.82 \mathrm{~g} / \mathrm{dL}, P=0.001$, and $3.73 \pm 0.29$ vs. $3.87 \pm 0.30$ $\mathrm{g} / \mathrm{dL}, P=0.001$, respectively). Although the normalized protein catabolic rate (nPCR) decreased from baseline after 24 months, the change was not significant (1.07 \pm 0.25 vs. $1.03 \pm 0.21 \mathrm{~g} / \mathrm{kg} / \mathrm{day}, P=0.433)$. Calcium and calcium-phosphorus products also increased significantly after OL-HDF therapy $(8.32 \pm 0.75$ vs. $8.75 \pm 0.80 \mathrm{mg} / \mathrm{dL}$,

Table 2. Twenty-four-month laboratory outcome changes in participants

\begin{tabular}{|c|c|c|}
\hline Variable & HD group $(n=135)$ & OL-HDF group $(n=44)$ \\
\hline \multicolumn{3}{|l|}{ Hemoglobin (g/dL) } \\
\hline Baseline & $10.61 \pm 1.15$ & $10.46 \pm 1.03$ \\
\hline After 24 months & $10.76 \pm 1.09$ & $11.08 \pm 0.82$ \\
\hline$P$ by paired $t$ test & 0.142 & 0.001 \\
\hline \multicolumn{3}{|l|}{$\mathrm{Ca}(\mathrm{mg} / \mathrm{dL})$} \\
\hline Baseline & $8.72 \pm 0.81$ & $8.32 \pm 0.75$ \\
\hline After 24 months & $8.19 \pm 0.93$ & $8.75 \pm 0.80$ \\
\hline$P$ by paired $t$ test & $<0.001$ & $<0.001$ \\
\hline \multicolumn{3}{|l|}{$P(m g / d L)$} \\
\hline Baseline & $4.88 \pm 1.35$ & $5.01 \pm 1.33$ \\
\hline After 24 months & $5.05 \pm 1.51$ & $5.52 \pm 1.46$ \\
\hline$P$ by paired $t$ test & 0.177 & 0.078 \\
\hline \multicolumn{3}{|l|}{$\operatorname{Ca} \times \mathrm{P}\left(\mathrm{mg}^{2} / \mathrm{dL}^{2}\right)$} \\
\hline Baseline & $42.58 \pm 12.42$ & $41.89 \pm 12.62$ \\
\hline After 24 months & $41.43 \pm 13.05$ & $50.80 \pm 20.79$ \\
\hline$P$ by paired $t$ test & 0.314 & 0.013 \\
\hline \multicolumn{3}{|l|}{ iPTH (pg/mL) } \\
\hline Baseline & $234.24 \pm 186.61$ & $300.10 \pm 178.80$ \\
\hline After 24 months & $354.27 \pm 293.11$ & $407.63 \pm 267.29$ \\
\hline$P$ by paired $t$ test & $<0.001$ & 0.008 \\
\hline \multicolumn{3}{|l|}{ nPCR (g/kg/day) } \\
\hline Baseline & $1.01 \pm 0.20$ & $1.07 \pm 0.25$ \\
\hline After 24 months & $0.98 \pm 0.18$ & $1.03 \pm 0.21$ \\
\hline$P$ by paired $t$ test & 0.165 & 0.433 \\
\hline \multicolumn{3}{|l|}{ Albumin (g/dL) } \\
\hline Baseline & $3.93 \pm 0.28$ & $3.73 \pm 0.29$ \\
\hline After 24 months & $3.88 \pm 0.32$ & $3.87 \pm 0.30$ \\
\hline$P$ by paired $t$ test & 0.051 & 0.001 \\
\hline
\end{tabular}

Data are presented as mean \pm standard variation.

$\mathrm{HD}$, hemodialysis; iPTH, intact-parathyroid hormone; nPCR, normalized protein catabolic rate; OL-HDF, online-hemodiafiltration. 
$P<0.001$ and $41.89 \pm 12.62$ vs. $50.80 \pm 20.79 \mathrm{mg}^{2} / \mathrm{dL}^{2}, P=$ 0.013 , respectively; Table 2).

We also investigated the effects of HDF conversion on anemia-related factors using multivariate adjustments (Table 3) [14]. Patients who converted to HDF showed significantly higher Hgb level than those who continued HD treatment without conversion $(0.2 \pm 1.3$ vs. $0.6 \pm 1.2$ $\mathrm{g} / \mathrm{dL}, P=0.010)$. These patients also exhibited marginally higher serum ferritin level and non-significantly lower transferrin saturation and erythropoiesis-stimulating agent doses.

To determine possible confounding effects of clinical factors, we conducted a linear regression analysis with multiple adjustments for changes in various laboratory values after HDF conversion (Table 4). Conversion to HDF resulted in elevation of serum calcium and albumin levels, both of which were significant even after adjustment for baseline demographics, inflammation, and dialysis duration.

\section{Discussion}

This study aimed to assess whether HDF treatment has a stronger influence on various parameters based on a multi-center cohort in Korea. We investigated changes in laboratory findings after a switch to OL-HDF over a

Table 3. Multiple linear regression on the changes of anemia-related variables after HDF conversion in hemodialysis patients

\begin{tabular}{|c|c|c|c|c|c|c|}
\hline \multirow{2}{*}{$\begin{array}{l}\text { Laboratory } \\
\text { measure }\end{array}$} & \multirow{2}{*}{ Patient groups } & \multirow{2}{*}{$\begin{array}{c}\text { Changes after } \\
\text { conversion }\end{array}$} & \multicolumn{2}{|l|}{ Univariate analysis } & \multicolumn{2}{|c|}{ Multivariate analysis $^{\mathrm{a}}$} \\
\hline & & & Unstandardized $\beta(95 \% \mathrm{Cl})$ & $P$ value & Unstandardized $\beta$ (95\% Cl) & $P$ value \\
\hline \multirow{2}{*}{$\begin{array}{l}\text { Hemoglobin } \\
(\mathrm{g} / \mathrm{dL})\end{array}$} & HD group & $0.2 \pm 1.3$ & Reference & & Reference & \\
\hline & OL-HDF group & $0.6 \pm 1.2$ & $0.47(0.04,0.90)$ & 0.034 & $0.74(0.18,1.30)$ & 0.010 \\
\hline \multirow[t]{2}{*}{ Ferritin (ng/mL) } & HD group & $93.2 \pm 239.9$ & Reference & & Reference & \\
\hline & OL-HDF group & $201.3 \pm 493.1$ & $108.1(-1.9,218.1)$ & 0.054 & $106.6(-7.1,220.2)$ & 0.066 \\
\hline \multirow[t]{2}{*}{ TSAT (\%) } & HD group & $-2.2 \pm 15.4$ & Reference & & Reference & \\
\hline & OL-HDF group & $-4.2 \pm 18.7$ & $-2.1(-7.6,3.5)$ & 0.467 & $3.4(-3.2,10.0)$ & 0.309 \\
\hline \multirow{2}{*}{$\begin{array}{l}\text { ESA dose } \\
\qquad(\mathrm{IU} / \mathrm{kg} / \mathrm{wk})^{\mathrm{b}}\end{array}$} & HD group & $-115.7 \pm 189.7$ & Reference & & Reference & \\
\hline & OL-HDF group & $-170.5 \pm 257.1$ & $-54.8(-126.1,16.5)$ & 0.131 & $-46.6(-119.1,25.9)$ & 0.206 \\
\hline
\end{tabular}

Data regarding changes in values are expressed as mean \pm standard deviation.

$\mathrm{Cl}$, confidence interval; ESA, erythropoiesis-stimulating agent; HD, hemodialysis; HDF, hemodiafiltration; OL-HDF, online-HDF; TSAT, transferrin saturation.

${ }^{a}$ Adjusted for age, sex, etiology of end-stage renal disease, time on dialysis, single-pool Kt/V, high sensitivity C-reactive protein (hs-CRP), use of ESA, and use of intravenous iron. ${ }^{b}$ For darbepoetin alfa and methoxy polyethylene glycol-epoetin beta, the dose per week was multiplied by 200 to convert the units from micrograms to international units (Ref. [14]).

Table 4. Multiple linear regression on the changes of serum mineral and albumin concentrations after HDF conversion in hemodialysis patients

\begin{tabular}{|c|c|c|c|c|c|c|}
\hline \multirow{2}{*}{$\begin{array}{l}\text { Laboratory } \\
\text { measure }\end{array}$} & \multirow{2}{*}{ Patient groups } & \multirow{2}{*}{$\begin{array}{c}\text { Changes after } \\
\text { conversion }\end{array}$} & \multicolumn{2}{|l|}{ Univariate analysis } & \multicolumn{2}{|c|}{ Multivariate analysis $^{a}$} \\
\hline & & & Unstandardized $\beta(95 \% \mathrm{Cl})$ & $P$ value & Unstandardized $\beta$ (95\% Cl) & $P$ value \\
\hline \multirow[t]{2}{*}{ Calcium (mg/dL) } & HD group & $-0.5 \pm 0.8$ & Reference & & Reference & \\
\hline & OL-HDF group & $0.4 \pm 0.7$ & $0.96(0.70,1.23)$ & $<0.001$ & $1.02(0.75,1.30)$ & $<0.001$ \\
\hline \multirow{2}{*}{$\begin{array}{l}\text { Phosphorus } \\
\text { (mg/dL) }\end{array}$} & HD group & $0.2 \pm 1.5$ & Reference & & Reference & \\
\hline & OL-HDF group & $0.5 \pm 1.9$ & $0.32(-0.23,0.88)$ & 0.253 & $0.52(-0.05,0.1 .08)$ & 0.072 \\
\hline \multirow[t]{2}{*}{$\operatorname{CaxP}\left(\mathrm{mg}^{2} / \mathrm{dL}^{2}\right)$} & HD group & $-1.2 \pm 13.2$ & Reference & & Reference & \\
\hline & OL-HDF group & $8.9 \pm 22.8$ & $10.1(4.6,15.6)$ & $<0.001$ & $12.1(6.5,17.7)$ & $<0.001$ \\
\hline \multirow[t]{2}{*}{ iPTH (pg/mL) } & HD group & $119 \pm 230$ & Reference & & Reference & \\
\hline & OL-HDF group & $107 \pm 258$ & $-11.7(-93.1,69.7)$ & 0.777 & $-35.7(-115.2,43.7)$ & 0.376 \\
\hline \multirow[t]{2}{*}{ Albumin (g/dL) } & HD group & $-0.1 \pm 0.3$ & Reference & & Reference & \\
\hline & OL-HDF group & $0.1 \pm 0.3$ & $0.20(0.09,0.30)$ & $<0.001$ & $0.19(0.08,0.30)$ & $<0.001$ \\
\hline
\end{tabular}

Data regarding the changes of the variables are expressed as mean \pm standard deviation.

$\mathrm{Cl}$, confidence interval; HD, hemodialysis; HDF, hemodiafiltration; iPTH, intact-parathyroid hormone; OL-HDF, online-HDF.

${ }^{a}$ Adjusted for age, sex, etiology of end-stage renal disease, time on dialysis, single-pool Kt/V, and high sensitivity C-reactive protein (hs-CRP). 
period of at least 12 months. Among the initial $179 \mathrm{HD}$ patients followed for more than 24 months, only 44 were switched to OL-HDF and were maintained for more than 12 months. According to a paired t-test to compare baseline and 24-month follow-up results, the level of Hgb increased significantly in patients after they were switched to OL-HDF. Although several studies reported no changes in anemia control in patients undergoing OL-HDF $[15,16]$, factors such as insufficient dialysis dose and short session time may have been responsible. In a recent crosssectional study, patients treated with OL-HDF enjoyed superior iron status, and their anemia control was superior to that of patients treated with regular HD [17]. In addition, Bonforte et al [18] reported that erythropoietin (EPO) supplementation and increased Hgb were stable after maintenance of OL-HDF for 24 months, although they only followed 14 patients for 24 months. As a possible explanation for these effects, Pedrini et al [19] suggested that high-efficiency convective OL-HDF reduced uremia-related derangement, which replenished iron stores and in turn lowered the necessary EPO dose and improved anemia [20].

The effect of OL-HDF on nutritional status remains obscure. Theoretically, OL-HDF has the disadvantage of albumin loss because of its high convective volume [21]. Orasan et al [22] reported that albumin was significantly lower in patients on OL-HDF compared with those on HD after follow-up for 6 and 12 months and concluded that OL-HDF did not improve nutritional status as assessed by albumin. Jean et al [23] also showed that serum albumin level was significantly lower during HDF periods when they observed patients during 3 incidental 6-month periods of HDF1-HD-HDF2. However, similar decreases in serum albumin also occurred in the HD group. A recent prospective controlled study that randomly assigned patients to either OL-HDF or high-flux HD did not find significantly decrease in serum albumin concentration in the OL-HDF group [12]. We also found no significant decrease in serum albumin levels in patients switched to OL-HDF. As serum albumin can be affected by other environmental factors, such as body fluid overload and inflammation $[24,25]$, these results suggest the safety and tolerability of OL-HDF in terms of nutritional status.

Measurement of nPCR is also used to determine nutritional status, because they reflect daily dietary protein intake in dialysis patients $[26,27]$. A previous prospective study that compared parameters between patients who were switched to HDF and those who remained on HD therapy showed that albumin and nPCR were significantly lower at 6 and 12 months in those who switched to HDF [22]. However, another study that compared parameters after 6 months of HD with parameters 6 months after a switch to HDF found no significant difference in nPCR level [23]. We also observed decreased level of nPCR in patients after they were switched to OL-HDF, but the change was not statistically significant. The nPCR is affected by residual renal function, and errors are possible depending on $\mathrm{Kt} / \mathrm{V}$, which is used to calculate $\mathrm{nPCR}$ [28]. Although multiple evaluations of nutritional conditions by other assessments would be necessary in these respects, our results suggest that OL-HDF has no injurious effect on the nutritional status of patients.

Few studies are available on the effects of OL-HDF on calcium-phosphate metabolism, but the relationship remains controversial. While some studies reported no differences in phosphate level between HD and HDF groups, others reported phosphate clearance during HDF [29-32]. However, the studies suffered from lack of data on protein intake or the result was inconsistent across baseline phosphate levels. A recent, multicenter, randomized controlled trial of chronic HD patients also found no significant change in serum phosphate, calcium, or iPTH between patient groups assigned to continue HD or switch to OL-HDF treatment [6]. In our study, phosphate level was higher after the switch to HDF, but the increase was not statistically significant. Although calcium phosphate product level was also elevated significantly in this group, the level was lower than the current recommended target value of $55 \mathrm{mg}^{2} / \mathrm{dL}^{2}$ [33]. Regarding concurrent significantly elevated albumin level, it is possible that elevated phosphate, calcium phosphate product, and iPTH levels are affected by improved oral intake and nutritional status. Further study on a larger sample of patients during a longer follow-up period is necessary to more clearly identify HDF effects on variables related to calcium-phosphate metabolism.

In Korea, the prevalence of ESRD has risen rapidly with the increase of the elderly population [34]. The proportion of ESRD patients on peritoneal dialysis has decreased while that of patients on HD has markedly increased in Korea [13,34]. According to a report from the Korean Society of Nephrology, only approximately $18 \%$ of 
the entire population of HD patients in 2016 underwent HDF therapy [35], mainly due to cost problems, and the cost difference between conventional HD and HDF has not been addressed. To apply OL-HDF therapy in spite of the additional costs and to implement this therapy effectively and economically, more evidence based on randomized control studies and reflecting positive effects of HDF in Korean dialysis patients is necessary [36].

The strength of this study is the initial differences in clinical conditions between the 2 study groups. At the time of initial recruitment, albumin level was significantly lower and dialysis duration was significantly shorter in the OL-HDF group, implying worse medical conditions than the other group. No injurious results on anemia and nutritional status were reported in the OL-HDF group, which could explain the effect of OL-HDF on improving anemia and nutrition. Although a study using a larger number of patients is needed, we suggest that changing dialysis modality to OL-HDF may improve clinical outcomes in dialysis patients.

There are also some limitations in this study. First, it had a small population. In addition, we did not investigate other parameters, such as quality of life and physical function, after switching to OL-HDF treatment. As no consensus exists on whether HDF can improve quality of life in ESRD patients, it would be meaningful to evaluate changes in this parameter. Although we investigated changes in parameters related to metabolic bone diseases (e.g., serum calcium, phosphate, and iPTH levels), we could not examine the change in vitamin $\mathrm{D}$ level. Being an observational cohort study, there were no specific criteria for selecting patients examined for change in HD to OL-HDF. OL-HDF is recommended in patients with uncontrolled hyperphosphatemia or those suffering from dialysis complications (e.g., beta 2-microglobulin amyloidosis and intradialytic complications). This could be applied to patients with a life span more than 5 years longer than that expected with renal function preservation or young patients that have not yet developed long-term complications that affect patient quality of life. Randomized controlled trials with a longer follow-up period are needed to provide more definite evidence of the beneficial effects of conversion from dialysis modality to HDF.

In conclusion, long-term OL-HDF treatment over 12 months or longer was associated with no detrimental effects on anemia or nutritional status. To investigate the effects of OL-HDF therapy on the Korean population, evaluating more parameters over a longer follow-up period with a larger number of patients is needed.

\section{Conflicts of interest}

All authors have no conflicts of interest to declare.

\section{Acknowledgments}

This study was supported by the Young Investigator Research Grant from the Korean Society of Nephrology (KSN 2018).

\section{Authors' contributions}

Yu Ho Lee and Yoon Soo Shin wrote the original manuscript; Yu Ho Lee, Yoon Soo Shin, and So-Young Lee participated in the data analysis and interpretation. Yang Gyun Kim, Sang Ho Lee, and Ju Young Moon participated in research ideation and study design; Hyeon Seok Hwang and Kyung Hwan Jeong performed statistical analysis; Shin Young Ahn prepared Figure 1; Hong Joo Lee and Dong-Young Lee prepared Tables 1 and 2; Eun-Jung Ko and Hye Jeong Cho prepared Tables 3 and 4; Dong Ho Yang and Hye Yun Jeong participated in supervision or mentorship. All authors read and approved the final manuscript.

\section{References}

[1] Chan CT, Blankestijn PJ, Dember LM, et al.; Conference Participants. Dialysis initiation, modality choice, access, and prescription: conclusions from a kidney disease: Improving Global Outcomes (KDIGO) Controversies Conference. Kidney Int 2019;96:37-47.

[2] Thomas B, Wulf S, Bikbov B, et al. Maintenance dialysis throughout the world in years 1990 and 2010. J Am Soc Nephrol 2015;26:2621-2633.

[3] Prasad N, Jha V. Hemodialysis in Asia. Kidney Dis (Basel) 2015;1:165-177.

[4] Liyanage T, Ninomiya T, Jha V, et al. Worldwide access to treatment for end-stage kidney disease: a systematic review. Lancet 2015;385:1975-1982.

[5] Nakai S, Iseki K, Tabei K, et al. Outcomes of hemodiafiltration based on Japanese dialysis patient registry. Am J Kid- 
ney Dis 2001;38(4 Suppl 1):S212-S216.

[6] Maduell F, Moreso F, Pons M, et al.; ESHOL Study Group. High-efficiency postdilution online hemodiafiltration reduces all-cause mortality in hemodialysis patients. J Am Soc Nephrol 2013;24:487-497.

[7] Ağbaş A, Canpolat N, Çalışkan S, et al. Hemodiafiltration is associated with reduced inflammation, oxidative stress and improved endothelial risk profile compared to high-flux hemodialysis in children. PLoS One 2018;13:e0198320.

[8] Suwabe T, Barrera-Flores FJ, Rodriguez-Gutierrez R, Ubara Y, Takaichi K. Effect of online hemodiafiltration compared with hemodialysis on quality of life in patients with ESRD: a systematic review and meta-analysis of randomized trials. PLoS One 2018;13:e0198320.

[9] Canaud B, Bragg-Gresham JL, Marshall MR, et al. Mortality risk for patients receiving hemodiafiltration versus hemodialysis: European results from the DOPPS. Kidney Int 2006;69:2087-2093.

[10] Vilar E, Fry AC, Wellsted D, Tattersall JE, Greenwood RN, Farrington K. Long-term outcomes in online hemodiafiltration and high-flux hemodialysis: a comparative analysis. Clin J Am Soc Nephrol 2009;4:1944-1953.

[11] Locatelli F, Karaboyas A, Pisoni RL, et al. Mortality risk in patients on hemodiafiltration versus hemodialysis: a 'realworld' comparison from the DOPPS. Nephrol Dial Transplant 2018;33:683-689.

[12] Morena M, Jaussent A, Chalabi L, et al.; FRENCHIE Study Investigators. Treatment tolerance and patient-reported outcomes favor online hemodiafiltration compared to highflux hemodialysis in the elderly. Kidney Int 2017;91:14951509.

[13] Lee HS, Ju YS, Song YR, et al. Current treatment status and medical costs for hemodialysis vascular access based on analysis of the Korean Health Insurance Database. Korean J Intern Med 2018;33:1160-1168.

[14] Macdougall IC, White C, Anker SD, et al.; PIVOTAL Investigators and Committees. Intravenous iron in patients undergoing maintenance hemodialysis. $N$ Engl J Med 2019;380:447-458.

[15] Lippi A, Rindi P, Baronti R, et al. Recombinant human erythropoietin and high flux haemodiafiltration. Nephrol Dial Transplant 1995;10 Suppl 6:51-54.

[16] Ward RA, Schmidt B, Hullin J, Hillebrand GF, Samtleben W. A comparison of on-line hemodiafiltration and highflux hemodialysis: a prospective clinical study. J Am Soc Nephrol 2000;11:2344-2350.
[17] Hamzagic N, Andjelkovic M, Pirkovic MS, Canovic P, Zaric $\mathrm{M}$, Petrovic D. Influence of dialysis modality on the treatment of anemia in patients with end-stage kidney disease. Ser J Exp Clin Res 2019 Jan 10 [Epub]. DOI: 10.2478/ sjecr-2018-0050.

[18] Bonforte G, Grillo P, Zerbi S, Surian M. Improvement of anemia in hemodialysis patients treated by hemodiafiltration with high-volume on-line-prepared substitution fluid. Blood Purif 2002;20:357-363.

[19] Pedrini LA, De Cristofaro V, Comelli M, et al. Long-term effects of high-efficiency on-line haemodiafiltration on uraemic toxicity. A multicentre prospective randomized study. Nephrol Dial Transplant 2011;26:2617-2624.

[20] Bowry SK, Gatti E. Impact of hemodialysis therapy on anemia of chronic kidney disease: the potential mechanisms. Blood Purif 2011;32:210-219.

[21] Vega A, Quiroga B, Abad S, et al. Albumin leakage in online hemodiafiltration, more convective transport, more losses? Ther Apher Dial 2015;19:267-271.

[22] Orasan RA, Patiu IM, Anghel D, et al. Variation of clinical and laboratory features in chronic dialysis patients treated with high-flux hemodialysis after switching to online hemodiafiltration. Int Urol Nephrol 2013;45:1415-1422.

[23] Jean G, Hurot JM, Deleaval P, Mayor B, Lorriaux C. Onlinehaemodiafiltration vs. conventional haemodialysis: a crossover study. BMC Nephrol 2015;16:70.

[24] Kubrusly M, Oliveira CM, Santos DC, Mota RS, Pereira ML. A comparative analysis of pre- and post-dialysis albumin as indicators of nutritional and morbi-mortality risks in haemodialysis patients. J Bras Nefrol 2012;34:27-35.

[25] Kaysen GA, Don BR. Factors that affect albumin concentration in dialysis patients and their relationship to vascular disease. Kidney Int Suppl 2003;(84):S94-S97.

[26] Lukowsky LR, Kheifets L, Arah OA, Nissenson AR, KalantarZadeh K. Nutritional predictors of early mortality in incident hemodialysis patients. Int Urol Nephrol 2014;46:129140.

[27] Fein PA, Weiss S, Avram MM, et al. Relationship of normalized protein catabolic rate with nutrition status and longterm survival in peritoneal dialysis patients. Adv Perit Dial 2015;31:45-48.

[28] Kanda E, Kato A, Masakane I, Kanno Y. A new nutritional risk index for predicting mortality in hemodialysis patients: Nationwide cohort study. PLoS One 2019;14:e0214524.

[29] Davenport A, Gardner C, Delaney M; Pan Thames Renal Audit Group. The effect of dialysis modality on phosphate 
control : haemodialysis compared to haemodiafiltration. The Pan Thames Renal Audit. Nephrol Dial Transplant 2010;25:897-901.

[30] Lornoy W, De Meester J, Becaus I, Billiouw JM, Van Malderen PA, Van Pottelberge M. Impact of convective flow on phosphorus removal in maintenance hemodialysis patients. J Ren Nutr 2006;16:47-53.

[31] Okuno S, Ishimura E, Kohno K, et al. Serum beta2-microglobulin level is a significant predictor of mortality in maintenance haemodialysis patients. Nephrol Dial Transplant 2009;24:571-577.

[32] Ok E, Asci G, Toz H, et al.; Turkish Online Haemodiafiltration Study. Mortality and cardiovascular events in online haemodiafiltration (OL-HDF) compared with high-flux dialysis: results from the Turkish OL-HDF Study. Nephrol Dial Transplant 2013;28:192-202.

[33] National Kidney Foundation. K/DOQI clinical practice guidelines for bone metabolism and disease in chronic kidney disease. Am J Kidney Dis 2003;42(4 Suppl 3):S1-S201.

[34] Jin DC. Current status of dialysis therapy in Korea. Korean J Intern Med 2011;26:123-131.

[35] Jin DC, Yun SR, Lee SW, et al. Current characteristics of dialysis therapy in Korea: 2016 registry data focusing on diabetic patients. Kidney Res Clin Pract 2018;37:20-29.

[36] Kim YW, Park S. Confronting practical problems for initiation of on-line hemodiafiltration therapy. Electrolyte Blood Press 2016;14:1-4. 